\title{
Liquefaction of $\mathrm{H}_{2}$ molecules upon exterior surfaces of carbon nanotube bundles
}

\author{
Sang Soo Han, Jeung Ku Kang, and Hyuck Mo Lee ${ }^{\text {a) }}$ \\ Department of Materials Science and Engineering, KAIST, Daejeon 305-701, Republic of Korea
}

Adri C. T. van Duin and William A. Goddard III

Materials and Process Simulation Center, California Institute of Technology, California 91125

(Received 9 December 2004; accepted 4 April 2005; published online 12 May 2005)

\begin{abstract}
We have used molecular dynamics simulations to investigate interaction of $\mathrm{H}_{2}$ molecules on the exterior surfaces of carbon nanotubes (CNTs): single and bundle types. At $80 \mathrm{~K}$ and $10 \mathrm{MPa}$, it is found that charge transfer occurs from a low curvature region to a high curvature region of the deformed CNT bundle, which develops charge polarization only on the deformed structure. The long-range electrostatic interactions of polarized charges on the deformed CNT bundle with hydrogen molecules are observed to induce a high local-ordering of $\mathrm{H}_{2}$ gas that results in hydrogen liquefaction. Our predicted heat of hydrogen liquefaction on the CNT bundle is $97.6 \mathrm{kcal} \mathrm{kg}^{-1}$. On the other hand, hydrogen liquefaction is not observed in the CNT of a single type. This is because charge polarization is not developed on the single CNT as it is symmetrically deformed under the same pressure. Consequently, the hydrogen storage capacity on the CNT bundle is much higher due to liquefaction than that on the single CNT. Additionally, our results indicate that it would also be possible to liquefy $\mathrm{H}_{2}$ gas on a more strongly polarized CNT bundle at temperatures higher than 80 K. (C) 2005 American Institute of Physics. [DOI: 10.1063/1.1929084]
\end{abstract}

In 1999, Ye et al. ${ }^{1}$ first reported an experimental finding that bundles of single-walled carbon nanotubes (SWCNTs) could absorb hydrogen in excess of $8 \mathrm{wt} \%$ at 100 bar and 80 $\mathrm{K}$. This result satisfies the target of the Department of Energy: namely that hydrogen fuel cell cars require a hydrogen capacity of 6.5 wt \% to match the range of a gasolinepowered car. Ye et al. ${ }^{1}$ suggested that when individual SWCNTs are separated at a pressure higher than 40 bar hydrogen is physisorbed on the exposed surfaces of the SWCNTs. We now report another conclusion. In general, gas molecules form a monolayer surface coverage on solids, ${ }^{2}$ as proved by the well-known Brunauer-Emmett-Teller (BET) theory of adsorption. ${ }^{3}$ When a surface is completely covered with a monolayer of adsorbate, additional molecules are adsorbed on the first layer of the adsorbate. However, the van der Waals interaction energy of the solid surface with the additional layers is negligible in comparison with the interaction energy of the molecules of the first layer, and it is therefore difficult for additional layers to form. Consequently, this BET theory indicates that the physisorption capacity of hydrogen at a constant temperature has no linear relationship with the gas pressure. ${ }^{4}$ In other words, the hydrogen physisorption capacity initially increases as the gas pressure increases, but then decreases. This phenomenon is called supercritical adsorption. ${ }^{4}$ However, Ye et al. ${ }^{1}$ experimentally showed that the hydrogen uptake capacity increases almost linearly with the increased hydrogen pressures. In addition, Gao et $a .^{5}$ reported that a single CNT with a cap shows a $\mathrm{H}_{2}$ uptake capacity of less than 2.18 wt \% hydrogen at $77 \mathrm{~K}$ and $10 \mathrm{MPa}$. This begs the question of why a high $\mathrm{H}_{2}$ uptake capacity can only be found in CNT bundles. We therefore examine how hydrogen physisorption behaves on

a) Author to whom all correspondence should be addressed; electronic mail: hmlee@kaist.ac.kr various surfaces of different CNT structures, particularly under conditions of high pressure and near liquid nitrogen temperature.

To study how hydrogen molecules interact with the surfaces of different CNT structures, we used molecular dynamics (MD) simulations with an isothermal-isobaric canonical ensemble condition. A time step of $0.5 \times 10^{-15} \mathrm{~s}$ was chosen to determine the equation of motions of atoms. The interatomic forces between hydrogen and carbon are based on a reactive force field (ReaxFF). ${ }^{6}$ We designed ReaxFF to reproduce the bond distances, energies, and charges of B3LYP (Becke three-parameter plus Lee-Yang-Parr functional) density functional theory (DFT) ${ }^{7}$ calculations for hydrocarbon systems. As shown in several studies, ${ }^{6,8-11}$ the ReaxFF accurately describes the breaking and formation of bonds, as well as atomic charges in polarized systems. To account for the van der Waals interactions between $\mathrm{C}-\mathrm{C}, \mathrm{C}-\mathrm{H}$, and $\mathrm{H}-\mathrm{H}$, ReaxFF relies on a distance-corrected Morse potential [Eqs. (1)-(3)], the fitted parameters of which have been reported in detail by Nielson et al. ${ }^{11}$ By including a shielded interaction [Eq. (2)], we avoided excessively high repulsions between bonded atoms (1-2 interactions) and atoms that share a valence angle (1-3 interactions). These interactions are expressed as follows:

$$
\begin{aligned}
E_{\mathrm{vdWaals}}= & \operatorname{Tap} \cdot D_{i j} \cdot\left\{\exp \left[\alpha_{i j} \cdot\left(1-\frac{f_{13}\left(r_{i j}\right)}{r_{\mathrm{vdW}}}\right)\right]\right. \\
& \left.-2 \cdot \exp \left[\frac{1}{2} \cdot \alpha_{i j} \cdot\left(1-\frac{f_{13}\left(r_{i j}\right)}{r_{\mathrm{vdW}}}\right)\right]\right\},
\end{aligned}
$$

$$
f_{13}\left(r_{i j}\right)=\left[r_{i j}^{p_{\mathrm{vdW} 1}}+\left(\frac{1}{\gamma_{\mathrm{vdW}}}\right)^{p_{\mathrm{vdW} 1}}\right]^{1 / p_{\mathrm{vdW} 1}},
$$




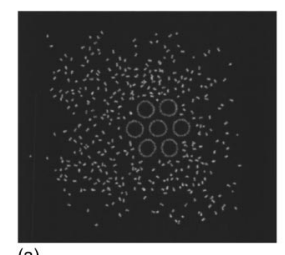

(a)

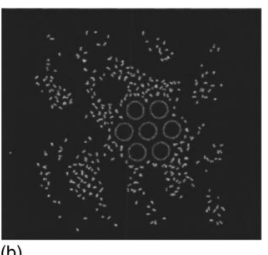

(b)
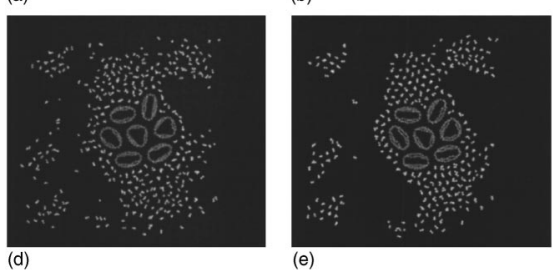

(e)

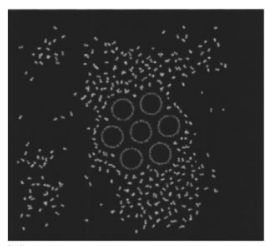
(c)

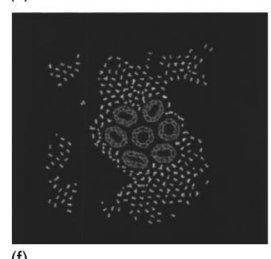

(f)

FIG. 1. Interactions of $\mathrm{H}_{2}$ molecules on the (5,5) SWCNT bundle surfaces at $80 \mathrm{~K}$ and $10 \mathrm{MPa}$. They are obtained from MD simulations employing the ReaxFF, where (a)-(f) indicate snapshots at 0, 2.5, 25, 27.5, 30, and $50 \mathrm{ps,}$ respectively. The grey color represents carbon atoms while the white one represents hydrogen.

$$
\operatorname{Tap}=\frac{20}{R_{\text {cut }}^{7}} \cdot r_{i j}^{7}-\frac{70}{R_{\text {cut }}^{6}} \cdot r_{i j}^{6}+\frac{84}{R_{\text {cut }}^{5}} \cdot r_{i j}^{5}-\frac{35}{R_{\text {cut }}^{4}} \cdot r_{i j}^{4}+1 .
$$

The term Tap in Eq. (3) is used to avoid energy discontinuity out of the nonbonded cutoff radius $\left(R_{\text {cut }}=10.0 \AA\right)$ of the ReaxFF. The terms in this polynomial were chosen to ensure that the first, second, and third derivatives of the nonbonded interactions to the distance were all continuous and went to zero at the cutoff boundary. This force field can therefore appropriately describe the weak van der Waals interaction, even for a long range of up to $10.0 \AA$. In using ReaxFF, we found that the physisorption energy of a $\mathrm{H}_{2}$ molecule on the exterior wall of a $(5,5)$ SWCNT is $-1.23 \mathrm{kcal} / \mathrm{mol}$, which falls in the physisorption energy range $(-0.2$ to $-2.0 \mathrm{kcal} / \mathrm{mol})$ on a graphene, calculated with several methods: the second-order Möller-Plesset perturbation theory; local density approximation, Slater exchange plus Vosko-Wilk-Nusair correlation; generalized gradient approximation, Becke exchange plus Lee-Yang-Parr correlation); and hybrid DFT (B3LYP) by Okamoto and Miyamoto. ${ }^{12}$ As a result, we can rely on ReaxFF to adequately explain the van der Waals interaction of hydrogen molecules on the CNT wall.

To model the CNT bundle, we used seven $(5,5)$ SWCNTs with a gap of $3.4 \AA$ between two of the SWCNT walls. The gap is similar to the graphite interplanar distance. In addition, to simulate how the $\mathrm{H}_{2}$ molecules interact with the CNT bundle, we used 840 carbon atoms and 400 hydrogen molecules. On the other hand, we used a $(10,10)$ SWCNT to model a single SWCNT and a $(5,5)$ at $(10,10)$ multiwalled CNT (MWCNT) to model a single MWCNT. The box size for all the MD simulations was $70 \AA \times 70 \AA$ $\times 4.66 \AA$, and the periodic boundary condition was applied. Because our simulation focused on the way $\mathrm{H}_{2}$ molecules interact with the exterior surfaces of the nanotubes, we did not explore how hydrogen molecules interact with carbon atoms inside the tube. During the MD simulations, we applied the pressure hydrostatically.

Figure 1 shows the MD simulation results on the behavior of hydrogen gas with a CNT bundle at $80 \mathrm{~K}$ and $10 \mathrm{MPa}$. The $\mathrm{H}_{2}$ molecules physically adsorb on the surfaces of the bundle at $2.5 \mathrm{ps}$ with a monolayer coverage and then come together with each other. The gathering degree of the hydroDownloaded 14 Dec 2005 to 131.215.225.171. Redistribution subject gen increases as the simulation time increases. At $27.5 \mathrm{ps}$, the SWCNT bundle is distorted under a high pressure of 10 $\mathrm{MPa}$. After $30 \mathrm{ps,}$ a high local-ordering of hydrogen is observed near the surfaces, indicating that the hydrogen liquefies. Furthermore, although not shown here, the total energy decreases abruptly between 27.5 and 30 ps. The enthalpy is a sum of the total internal energy for all the atoms and the external energy of $P \Delta V$, where $P$ is the applied pressure and $\Delta V$ is the volume change. Because the $P \Delta V$ term in our simulation is negligible compared to the internal energy, the enthalpy can be approximated to the internal energy. Consequently, the predicted energy difference of $97.6 \mathrm{kcal} / \mathrm{kg}$ between 27.5 and 30 ps can be considered as the latent heat of vaporization, which is consistent with the experimental value of $108.0 \mathrm{kcal} / \mathrm{kg} .{ }^{13}$ Ye et al. ${ }^{1}$ suggested that the CNT bundle would expand under a high pressure and that the individual SWCNTs should be separated. However, in our MD simulation, the bundle does not appear to expand but rather to contract, and no $\mathrm{H}_{2}$ molecule was adsorbed among the SWCNT bundle. As shown by Lawrence and $\mathrm{Xu},{ }^{14}$ the resistance measurement from a purified SWCNT bundle at room temperature reveals that the bundle was compressed under a high external pressure. This leads to a slight increase in conductivity when the pressure is increased. During the simulation, we also observed no chemisorption of hydrogen gas on the CNT surface. According to our DFT calculation, ${ }^{15}$ the chemisorption reaction is endothermic by $18.4 \mathrm{kcal} / \mathrm{mol}$, and its high transition state barrier of $78.3 \mathrm{kcal} / \mathrm{mol}$ indicates that there is a kinetic difficulty that may prevent chemisorption from taking place. From these results, we conclude that the increased $\mathrm{H}_{2}$ uptake capacity on the CNT bundle at $80 \mathrm{~K}$ and $10 \mathrm{MPa}$ can be attributed to the liquefaction of the $\mathrm{H}_{2}$ gas. Our results also account for other phenomena reported by Ye et al. ${ }^{1}$ For instance, the kink that occurs at around $4 \mathrm{MPa}$ and the steep slope of the hydrogen adsorption isotherm at pressures between 4 and $10 \mathrm{MPa}$. From the MD simulations, we observe that the liquefaction of $\mathrm{H}_{2}$ molecules on the CNT bundle occurs at pressures higher than $2 \mathrm{MPa}$ at $80 \mathrm{~K}$. At pressures lower than $2 \mathrm{MPa}$, the $\mathrm{H}_{2}$ molecules are physically adsorbed on the CNT bundle with a monolayer coverage. We also investigated the hydrogen-adsorption behavior on the CNT bundle at $300 \mathrm{~K}$ and $10 \mathrm{MPa}$. After $50 \mathrm{ps}$, the CNT bundle and hydrogen molecules resemble their initial geometries. The $\mathrm{H}_{2}$ molecules fail to penetrate the interstices of the tubes but wander on the exterior surfaces of the tubes. This phenomenon supports the experimental observation of Laurence and $\mathrm{Xu}^{14}$ that the hydrogen adsorption capacity is very low at $300 \mathrm{~K}$ and $10 \mathrm{MPa}$. They showed that only 0.6 wt \% hydrogen molecules adsorb on the CNT bundle at 294 $\mathrm{K}$ and $10 \mathrm{MPa}$.

We also examined whether the liquefaction of $\mathrm{H}_{2}$ molecules can occur on a single CNT. Figure 2 represents MD simulations of $\mathrm{H}_{2}$ molecules interacting with the surfaces of a single SWCNT and a MWCNT at $80 \mathrm{~K}$ and $10 \mathrm{MPa}$. After $50 \mathrm{ps}$, no liquefaction of $\mathrm{H}_{2}$ molecules is found. In these cases, the enthalpy change that signals a phase transition from gas to liquid is not observed. Furthermore, Figs. 2(c) and 2(f) show that when $\mathrm{H}_{2}$ is about $2.9 \AA$ from the CNT surface in the early simulation period the $\mathrm{H}_{2}$ molecules adsorb on the SWCNT and MWCNT surfaces with a monolayer coverage. As with the bundle, the CNT in these cases is distorted under a high pressure. Moreover, because $\mathrm{H}_{2}$ molecules adsorb on the single CNT surface at $80 \mathrm{~K}$ irrespective to AIP license or copyright, see http://apl.aip.org/apl/copyright.jsp 

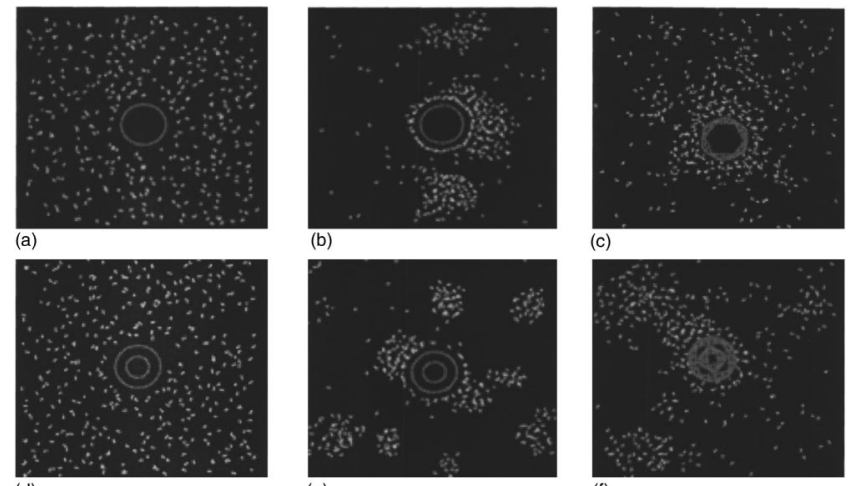

(d)

(e)

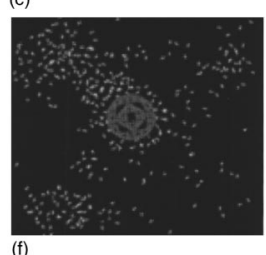

FIG. 2. Interactions of $\mathrm{H}_{2}$ molecules on the single $(10,10)$ SWCNT and the single $(5,5)$ at $(10,10)$ MWCNT at $80 \mathrm{~K}$ and $10 \mathrm{MPa}$. They are obtained from MD simulations employing the ReaxFF, where (a)-(c) are for the SWCNT and (d)-(f) for the MWCNT. (a) and (d) are taken at 0 ps, (b) and (e) at $10 \mathrm{ps}$, and (c) and (f) at $50 \mathrm{ps}$. The grey and white colors represent carbon atoms and hydrogen atoms, respectively.

of pressures, $\mathrm{H}_{2}$ adsorption capacities on the single SWCNT and the single MWCNT are computed to be lower than that of the CNT bundle, which was proven by the previous work. ${ }^{5}$

Remarkably, the liquefaction of $\mathrm{H}_{2}$ molecules occurs only with a CNT bundle, and not with a single SWCNT or a single MWCNT. Of all the MD simulation data on the interactions of $\mathrm{H}_{2}$ molecules with single and bundle types, the only noticeable difference occurs on the structures of the CNTs distorted under a high pressure. The SWCNT bundle deforms into oval shapes under high pressure, as shown in Fig. 1(f), while the shapes of the single SWCNT and MWCNT structures are almost symmetrical, as shown in Figs. 2(c) and 2(f). A recent DFT study ${ }^{16}$ showed that if the SWCNT deforms into an oval shape the electron charges transfer from a low-curvature region to a high-curvature region. This charge transfer on the SWCNT bundle develops polarized charges on the CNT structures. Therefore, when the CNT deforms into the oval shape, the electrostatic interaction between the polarized charges on the deformed nanotubes and the induced quadruple charge moments on the hydrogen molecules could result in a high ordering of hydrogen molecules. In a single SWCNT and MWCNT, the phenomenon of the charge transfer is not observed due to the symmetrically deformed shapes. Thus, the local ordering of hydrogen is not observed. This finding has also been supported by a study of Simonyan et al.,${ }^{17}$ who used a grand canonical Monte Carlo simulation to investigate the adsorption of hydrogen gas on the charged CNT. Their study showed that $\mathrm{H}_{2}$ molecules can be adsorbed on charged CNT surfaces due to the interactions between the monopole charges on the CNT and the induced charges on the $\mathrm{H}_{2}$. On the other hand, the long-range electrostatic interaction between the dipole charges on deformed SWCNTs and the induced quadruple charge moments on the $\mathrm{H}_{2}$ is considered to be responsible for aligning the adsorbed hydrogen along the locally ordered CNT structures. This process eventually makes the hydrogen molecules condense into a liquid state.

In summary, we observed an interesting phenomenon in which hydrogen gas molecules transformed into a liquid phase at around $80 \mathrm{~K}$ and $10 \mathrm{MPa}$ on the surfaces of a CNT bundle due to the long-range electrostatic interaction with the deformed, oval-shaped CNT. Liquid hydrogen has good potential as a hydrogen fuel for vehicular transport because it has a high mass density $\left(70.8 \mathrm{~kg} \mathrm{~m}^{-3}\right)$ and is relatively safe. Despite these advantages, the liquefaction of hydrogen is expensive and requires an intensive cryogenic process for cooling due to the low condensation temperature $(21.2 \mathrm{~K}$ at 1 bar). ${ }^{13}$ On the other hand, the high liquefaction temperature of hydrogen at around $80 \mathrm{~K}$ on a moderately polarized CNT bundle implies that $\mathrm{H}_{2}$ gas could be liquefied on a more strongly polarized CNT bundle at the temperatures higher than $80 \mathrm{~K}$.

This research was supported by a grant (Code No. 04K1501-02210) from "Center for Nanostructured Materials Technology" under "21st Century Frontier R\&D Programs" of the Ministry of Science and Technology, Korea.

${ }^{1}$ Y. Ye, C. C. Ahn, C. Witham, B. Fultz, J. Liu J, A. G. Rinzler, D. Colbert, K. A. Smith, and R. E. Smalley, Appl. Phys. Lett. 74, 2307 (1999).

${ }^{2}$ B. A. Beebe, J. Biscoe, W. R. Smith, and C. B. Wendell, J. Am. Chem. Soc. 69, 95 (1947).

${ }^{3}$ S. Brunauer, P. H. Emmett, and E. Teller, J. Am. Chem. Soc. 60, 309 (1938).

${ }^{4}$ Y. Zhou, K. Feng, Y. Sun, and L. Zhou, Chem. Phys. Lett. 380, 526 (2003).

${ }^{5}$ H. Gao, X. B. Wu, J. T. Li, G. T. Wu, J. Y. Lin, K. Wu, and D. S. Xu, Appl. Phys. Lett. 83, 3389 (2003).

${ }^{6}$ A. C. T. van Duin, S. Dasgupta, F. Lorant, and W. A. Goddard III, J. Phys. Chem. A 105, 9396 (2001).

${ }^{7}$ A. D. Becke, J. Chem. Phys. 98, 5648 (1993).

${ }^{8}$ A. C. T. van Duin, A. Strachan, S. Stewman, Q. Zhang, X. Xu, and W. A. Goddard III, J. Phys. Chem. A 107, 3803 (2003).

${ }^{9}$ A. Strachan, A. C. T. van Duin, D. Chakraborty, S. Dasgupta, and W. A. Goddard III, Phys. Rev. Lett. 91, 098301 (2003).

${ }^{10}$ Q. Zhang, T. Çağin, A. C. T. van Duin, W. A. Goddard III, Y. Qi, and L. G. Hector, Phys. Rev. B 69, 045423 (2004).

${ }^{11}$ K. D. Nielson, A. C. T. van Duin, J. Oxgaard, W.-Q. Deng, and W. A. Goddard III, J. Phys. Chem. A 109, 493 (2005).

${ }^{12}$ Y. Okamoto and Y. Miyamoto, J. Phys. Chem. B 105, 3470 (2001).

${ }^{13}$ L. Zhou, Renew. Sust. Energ. Rev. 9, 35 (2005).

${ }^{14}$ J. Lawrence and G. Xu, Appl. Phys. Lett. 84, 918 (2004).

${ }^{15}$ S. S. Han and H. M. Lee, Carbon 42, 2169 (2004).

${ }^{16}$ S. B. Fagan, R. Mota, A. J. R. de Silva, and A. Fazio, Nano Lett. 4, 975 (2004).

${ }^{17}$ V. V. Simonyan, P. Diep, and J. K. Johnson, J. Chem. Phys. 111, 9778 (1999). 